\title{
A weakly nonlinear Alfvénic pulse in a transversely inhomogeneous medium
}

\author{
D. Tsiklauri, T. D. Arber, and V. M. Nakariakov
}

Physics Department, University of Warwick, Coventry, CV4 7AL, UK

Received 31 July 2001 / Accepted 24 September 2001

\begin{abstract}
The interaction of a weakly nonlinear Alfvénic pulse with an Alfvén speed inhomogeneity in the direction perpendicular to the magnetic field is investigated. Identical to the phase mixing experienced by a harmonic Alfvén wave, sharp transverse gradients are generated in the pulse by the inhomogeneity. In the initial stage of the evolution of an initially plane Alfvénic pulse, the transverse gradients efficiently generate transversely propagating fast magnetoacoustic waves. However, high resolution full MHD numerical simulations of the developed stage of the pulse evolution show that the generation saturates due to destructive wave interference. It is shown that the weakly non-linear description of the generated fast magnetoacoustic wave is well described by the driven wave equation proposed in Nakariakov et al. (1997), and a simple numerical code (2D MacCromack), which solves it with minimal CPU resources, produces identical results to those obtained from the full MHD code (Lare2d, Arber et al. 2001). A parametric study of the phenomenon is undertaken, showing that, contrary to one's expectations, steeper inhomogeneities of the Alfvén speed do not produce higher saturation levels of the fast wave generation. There is a certain optimal gradient of the inhomogeneity that ensures the maximal efficiency of the fast wave generation.
\end{abstract}

Key words. magnetohydrodynamics (MHD) - waves - Sun: activity - Sun: corona

\section{Introduction}

Many astrophysical objects and space plasma systems are significantly influenced by magnetohydrodynamic (MHD) waves. MHD waves constitute the basis of many current theories of solar and stellar wind acceleration, the heating of solar and stellar coronae and the stability of molecular clouds, etc. In solar physics interest in MHD waves has increased significantly with the successful detection of MHD waves in coronal loops and plumes by recent observations from the SOHO and TRACE spacecrafts. This, in turn, has generated lively interest in MHD waves in the theoretical solar physics community in the context of coronal plasma heating and solar wind acceleration (see Roberts 2000 for a review).

A significant aspect in MHD wave dynamics, which has attracted considerable attention recently, is the interaction of waves with plasma inhomogeneities. Because of the essentially anisotropic nature of MHD waves, they are affected by the inhomogeneities in many ways. In particular, structuring of the medium leads to the appearance of long wave-length dispersion, the existence of local resonances associated with e.g. resonant absorption, and

Send offprint requests to: D. Tsiklauri, e-mail: tsikd@astro.warwick.ac.uk
Alfvén wave phase mixing (see, e.g. Roberts 1991; Roberts \& Ulmschneider 1997).

In the majority of astrophysical applications, almost incompressible Alfvén waves are subject to very weak dissipation. Therefore, physical mechanisms which can create small scales in the waves, enhancing the dissipation, are very important. One of these mechanisms is phase mixing of Alfvén waves interacting with a plasma inhomogeneity. When an initially plane Alfvén wave propagates along a straight magnetic field, the presence of an Alfvén speed inhomogeneity across the field leads to distortion of the wave front. In other words, each magnetic interface supports Alfvén waves propagating with its own local Alfvén speed. After a while, the neighbouring magnetic interfaces are oscillating out of phase and the Alfvén wave becomes "phase-mixed". This phenomenon dramatically decreases the perpendicular wavelength and generates very sharp transverse gradients. In the presence of finite viscosity or resistivity, the short wavelengths are subject to enhanced dissipation. Since this mechanism was proposed by Heyvaerts \& Priest (1983) for heating the solar corona, the linear regime of Alfvén wave phase mixing has been intensively investigated by many researchers, mainly in the coronal heating context (Abdelatif 1987; Browning 1991; Ireland \& Priest 1997; see also the critical paper of Parker 1991), including investigation of Alfvén wave 
phase mixing in inhomogeneous steady flows (Ryutova \& Habbal 1995; Nakariakov et al. 1998) and in two dimensional structures (Hood et al. 1997; Ruderman et al. 1998; Ruderman et al. 1999; De Moortel et al. 1999; De Moortel et al. 2000) and studies of secondary Kelvin-Helmholtz instabilities (Browning \& Priest 1984). Possible manifestations of this effect in coronal observations have been discussed (Ireland 1996; De Moortel \& Hood 2000) and numerical simulations of Alfvén wave phase mixing have been undertaken in various astrophysical and geophysical contexts (Botha et al. 2000; Grappin et al. 2000; Malara et al. 1996; Ofman \& Davila 1997).

In the linear regime of Alfvén wave phase mixing, the wave does not interact with other wave modes, and the wave dissipation is enhanced due to the generation of small scales across the field. In the nonlinear regime, Alfvén waves interact with, in particular, magnetoacoustic waves. For the Alfvén wave amplitudes expected in the lower corona of the Sun, the linear treatment of the process of phase mixing seemed to be justified (e.g. Sakurai \& Granik 1984) as the Alfvén waves are subject to cubic nonlinearity, not quadratic as for magnetoacoustic waves. The presence of structuring can dramatically affect the efficiency of nonlinear processes. In coronal holes, outwardly propagating Alfvén waves are amplified by stratification and, therefore, quickly reach the nonlinear regime (Ofman \& Davila 1997; Nakariakov et al. 2000). In the presence of structuring across the field, phase mixing leads to nonlinear generation of transversely propagating fast magnetoacoustic waves (Nakariakov et al. 1997, 1998).

The nonlinear generation of fast waves, based upon a combination of inhomogeneous and nonlinear effects, can provide new physical mechanisms for MHD wave dissipation. In the linear regime of phase mixing, Alfvén waves dissipate due to the shear component of the viscosity tensor, while the fast magnetoacoustic waves dissipate due to the bulk viscosity. In the nearly collisionless coronal plasma the bulk viscosity is estimated to be $10^{10}$ times larger than the shear viscosity. Thus, fast magnetoacoustic waves that are generated by Alfvén waves dissipate much more efficiently than the Alfvén waves. This mechanism can be regarded as the indirect heating of plasma by phase mixing.

In the corona of the Sun there is some evidence that the shear viscosity may actually be of the same order as the bulk viscosity (Nakariakov et al. 1999). In this case, the mechanism of indirect heating is still very important, because it provides a possibly of heat distribution across the magnetic field. Indeed, the transversely propagating fast waves carry the energy across the field and deposit it at some distance from the region of phase mixing. Regular thermal conduction is depressed in the perpendicular direction by the magnetic field. Thus, the mechanism of indirect heating of astrophysical plasmas by Alfvén wave phase mixing requires serious investigation.

Nakariakov et al. (1997), by applying a weakly nonlinear description for the dynamics of the generated fast magnetoacoustic waves, pointed out that phase mixed Alfvén waves had to generate secularly growing fast waves (their Eq. (28)). This analytical result was successfully reproduced in full-MHD numerical simulations of the initial stage of the fast wave generation. Botha et al. (2000) undertook numerical simulations of this phenomenon over much longer times, investigating the efficiency of the indirect heating by considering harmonic phase-mixed Alfvén perturbations. They found that initially the amplitude of the generated fast magnetoacoustic waves grows but then saturates and does not reach a substantial fraction of the Alfvén wave amplitude. They further postulated that this saturation was due to the nature of the source term for fast waves. For harmonic Alfvén waves, as distinct from the single pulse considered in this paper, phase mixing leads to there being many fast wave sources in the direction of the background inhomogeneity. Indeed, the number of such sources, and their spacing, changes in time and as a consequence the fast wave sources cannot remain coherent and destructive wave interference limits the growth of fast wave energy.

In this work, we simulate numerically the interaction of a linear Alfvénic pulse with a one-dimensional, perpendicular to the magnetic field, plasma inhomogeneity. The pulse phase mixes, leading to steep transverse gradients. This leads to the generation of fast magnetoacoustic waves. This work has three objectives: to investigate whether a phase-mixed Alfvénic pulse, as opposed to Botha et al.'s (2000) harmonic Alfvén wave, can efficiently generate fast magnetoacoustic waves; to check whether the rapid growth (in time) of the generated fast magnetoacoustic waves still holds at the later stages and to clarify the role of the destructive wave interference for an isolated pulse.

We would like to emphasize that in this work we consider only small amplitude (relative amplitude normalized to the unperturbed value $A=0.001$ ) Alfvén waves in a low beta plasma. Such a physical situation is relevant to the lower corona of the Sun where the observed amplitudes of the Alfvén waves are indeed small. The more general case of large amplitudes $(A \sim 1)$ and arbitrary plasma beta, which is relevant e.g. for the Alfvén waves in the solar wind, will be reported elsewhere (Tsiklauri et al. 2001).

The paper is organized as follows: in Sect. 2 we outline the basic aspects of our model. Section 3 presents the results of our numerical calculation. In Sect. 4 we close with a brief discussion of the main results of this paper.

\section{The model}

In our model we use equations of cold (zero plasma- $\beta$ ) ideal MHD

$\rho \frac{\partial \boldsymbol{V}}{\partial t}+\rho(\boldsymbol{V} \cdot \nabla) \boldsymbol{V}=-\frac{1}{4 \pi} \boldsymbol{B} \times \operatorname{curl} \boldsymbol{B}$,

$\frac{\partial \boldsymbol{B}}{\partial t}=\operatorname{curl}(\boldsymbol{V} \times \boldsymbol{B})$,

$\operatorname{div} \boldsymbol{B}=0$, 


$$
\frac{\partial \rho}{\partial t}+\operatorname{div}(\rho \boldsymbol{V})=0
$$

where $\boldsymbol{B}$ is the magnetic field, $\boldsymbol{V}$ is plasma velocity, and $\rho$ is plasma mass density.

We consider Eqs. (1)-(4) in Cartesian coordinates $(x, y, z)$ and for simplicity assume that there is no variation in the $y$-direction, i.e. $(\partial / \partial y=0)$. The equilibrium state is taken to be an inhomogeneous plasma of density $\rho_{0}(x)$ and a uniform magnetic field $B_{0}$ in the $z$-direction.

Nakariakov et al. (1997) have obtained a set of governing equations for the finite amplitude perturbations for the above formulated physical system (see, also, Nocera et al. 1986), and for brevity we do not repeat their derivation here. However, we quote their final analytic result, which is two coupled, non-linear wave equations describing nonlinear coupling of Alfvén and fast magnetoacoustic waves

$$
\begin{gathered}
\frac{\partial^{2} V_{y}}{\partial t^{2}}-C_{\mathrm{A}}^{2}(x) \frac{\partial^{2} V_{y}}{\partial z^{2}}=\frac{1}{\rho_{0}(x)}\left(\frac{\partial N_{2}}{\partial t}+\frac{B_{0}}{4 \pi} \frac{\partial N_{5}}{\partial z}\right), \\
\frac{\partial^{2} V_{x}}{\partial t^{2}}-C_{\mathrm{A}}^{2}(x)\left(\frac{\partial^{2} V_{x}}{\partial x^{2}}+\frac{\partial^{2} V_{x}}{\partial z^{2}}\right)= \\
\frac{1}{\rho_{0}(x)}\left(\frac{\partial N_{1}}{\partial t}+\frac{B_{0}}{4 \pi} \frac{\partial N_{4}}{\partial z}-\frac{B_{0}}{4 \pi} \frac{\partial N_{6}}{\partial x}\right),
\end{gathered}
$$

where $C_{\mathrm{A}}(x)=B_{0} / \sqrt{4 \pi \rho_{0}(x)}$ is a local Alfvén speed and following notation has been used

$$
\begin{gathered}
N_{1}=-\rho \frac{\partial V_{x}}{\partial t}-\left(\rho_{0}(x)+\rho\right)\left(V_{x} \frac{\partial V_{x}}{\partial x}+V_{z} \frac{\partial V_{x}}{\partial z}\right) \\
+\frac{B_{z}}{4 \pi}\left(\frac{\partial B_{x}}{\partial z}-\frac{\partial B_{z}}{\partial x}\right)-\frac{B_{y}}{4 \pi} \frac{\partial B_{y}}{\partial x}, \\
N_{2}=-\rho \frac{\partial V_{y}}{\partial t}-\left(\rho_{0}(x)+\rho\right)\left(V_{x} \frac{\partial V_{y}}{\partial x}+V_{z} \frac{\partial V_{y}}{\partial z}\right) \\
\quad+\frac{B_{x}}{4 \pi} \frac{\partial B_{y}}{\partial x}+\frac{B_{z}}{4 \pi} \frac{\partial B_{y}}{\partial z}, \\
N_{4}=-\frac{\partial}{\partial z}\left(V_{z} B_{x}-V_{x} B_{z}\right), \\
N_{5}=-\frac{\partial}{\partial z}\left(V_{z} B_{y}-V_{y} B_{z}\right)+\frac{\partial}{\partial x}\left(V_{y} B_{x}-V_{x} B_{y}\right), \\
N_{6}=\frac{\partial}{\partial x}\left(V_{z} B_{x}-V_{x} B_{z}\right) .
\end{gathered}
$$

As noted in Nakariakov et al. 1997, the non-linear dynamics of Alfvén waves is described by Eq. (5). Equation (6) is the governing equation for the non-linear dynamics of finite amplitude fast magnetoacoustic waves. In the linear limit when the right-hand-side terms in the Eqs. (5) and (6) tend to zero, Alfvén and fast magnetoacoustic waves propagate along the applied magnetic field independently. Therefore, in this case Eq. (5) describes phasemixed Alfvén waves, which propagate along $z$-axis perturbing physical quantities in the $x O z$ plane. Equation (6) describes either trapped or leaking fast magnetoacoustic waves (depending whether there is a maximum or minimum in the density profile $\left.\rho_{0}(x)\right)$.

We consider the physical situation when initially fast magnetoacoustic perturbations are absent and the initial amplitude of the Alfvén wave is small. In this case, if the Alfvén wave is initially a plane wave, the subsequent evolution of the wave, due to the difference in local Alfvén speed across the $x$-coordinate, leads to the distortion of the wave front. Hence the appearance of transverse (with respect to the applied magnetic field) gradients, which grow linearly with time. Therefore the leading non-linear term on the right-hand-side of Eq. (6) is the transverse gradient of the magnetic pressure perturbation, i.e. to a fairly good accuracy (which is substantiated by our numerical calculations presented below) the dynamics of the fast magnetoacoustic waves can be described by an approximate equation (Nakariakov et al. 1997)

$$
\begin{aligned}
\frac{\partial^{2} V_{x}}{\partial t^{2}}-C_{\mathrm{A}}^{2}(x) & \left(\frac{\partial^{2} V_{x}}{\partial x^{2}}+\frac{\partial^{2} V_{x}}{\partial z^{2}}\right)= \\
& -\frac{1}{4 \pi \rho_{0}(x)} \frac{\partial}{\partial t}\left(B_{y} \frac{\partial B_{y}}{\partial x}\right) .
\end{aligned}
$$

\section{Numerical results}

As in the work of Botha et al. (2000), we have used the following background density profile

$\rho_{0}(x)=3-2 \tanh (\lambda x)$.

Here, $\lambda$ is a free parameter which controls the steepness of the density profile gradient. In our normalization, which is the same as that of Botha et al. (2000), the local Alfvén speed is $C_{\mathrm{A}}(x)=1 / \sqrt{3-2 \tanh (\lambda x)}$, and we plot it in Fig. 1 for the parameters used in our numerical simulations (see below). As can be seen from the plot, the local Alfvén speed has a gradient at $x=0$ and its value is controlled by the $\lambda$-parameter. Full numerical calculation of the non-linear set of MHD Eqs. (1)-(4), in the abovementioned geometry, has been performed using Lare2d (Arber et al. 2001). Lare2d is a numerical code which operates by taking a Lagrangian predictor-corrector time step and after each Lagrangian step all variables are conservatively re-mapped back onto the original Eulerian grid using Van Leer gradient limiters. This code was also used to produce the results in Botha et al. (2000).

We set up the code in such a way that initially fast magnetoacoustic perturbations are absent and the initial amplitude of the Alfvén pulse is small, i.e. $A=0.001$. In all our numerical runs plasma $\beta$ was fixed at 0.001 . In the numerical simulations the Alfvén perturbation is initially a plane (with respect to the $x$-coordinate) pulse, which has a Gaussian structure in the $z$-coordinate, and is moving at the local Alfvén speed $C_{\mathrm{A}}(x)$ :

$B_{y}(x, z, t)=A \exp \left(-\frac{\left(z-C_{\mathrm{A}}(x) t\right)^{2}}{\delta}\right)$.

Here, $\delta$ is a free parameter which controls the width of the initial Gaussian Alfvén pulse. The simulation box size is 


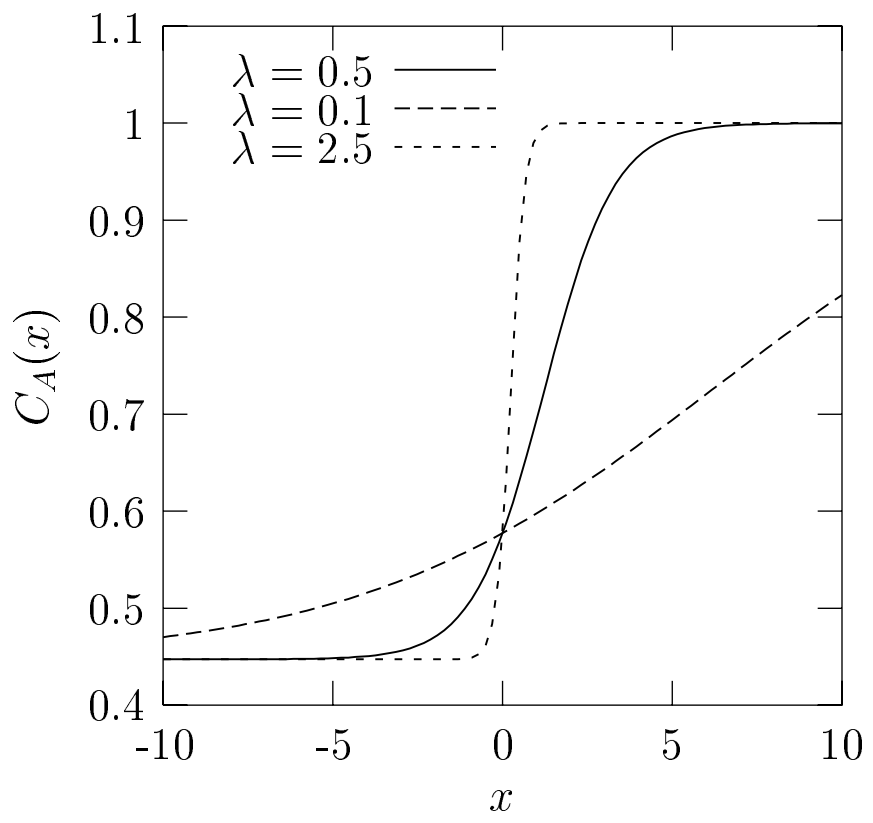

Fig. 1. Dimensionless Alfvén speed $C_{\mathrm{A}}(x)$ versus $x$ for $\lambda=0.5$ solid line, $\lambda=0.1$ long dashed line, $\lambda=2.5$ short dashed line.

set by the limits $-17.5<x<17.5$ and $-27.5<z<27.5$ and the pulse starts to move from point $z=-25.0$ towards the positive $z$ 's. We have performed calculation on various resolutions in order to achieve convergence of the results. The graphical results presented here are for the spatial resolution $4400 \times 1400$, which refers to number of grid points in $z$ and $x$ directions respectively. In Fig. 2 we show how the initial pulse $\left(B_{y}\right)$ has evolved by $t=10$. It can be seen from the graph that because of the difference in local Alfvén speeds (recall from Fig. 1 that for $x<0, C_{\mathrm{A}}(x)=1 / \sqrt{5}$ and for $\left.x>0, C_{\mathrm{A}}(x)=1\right)$ the initially flat (with respect to $x$-coordinate) Gaussian pulse has been distorted along the $x=0$ axis. This distortion of the pulse front creates a transverse gradient $\left(\partial B_{y} / \partial x\right)$ which according to Eq. (12) is a driving force for the generation of a fast magnetoacoustic wave $\left(V_{x}\right)$. At time $t=0$ the fast magnetoacoustic wave was absent from the system. While at time $t=10$ we see from Fig. 3 that $V_{x}$ has grown to the square of the initial Alfvén perturbation amplitude i.e. $10^{-6}$.

Figure 4 presents a contour plot of $B_{y}$ at time $t=50$. We observe that the Alfvén pulse has been distorted even further, thus creating even stronger transverse gradients which drive the growth of fast magnetoacoustic waves. In Fig. 5 we plot the generated $V_{x}$ at the same time, and we observe that its maximal value has grown further to $1.78 \times 10^{-6}$.

We have kept track of the maximal value of generated fast magnetoacoustic wave, $V_{x}^{a}(t)=\max \left(\left|V_{x}(x, z, t)\right|\right)$, as the simulation progresses. The results are presented by the thick solid line in Fig. 6. As we mentioned above, at the early stages of evolution, the dynamics of the generated fast magnetoacoustic wave is governed by Eq. (12). In order to prove this, we have written a code which, instead of solving the fully non-linear set of MHD Eqs. (1)-(4), solves

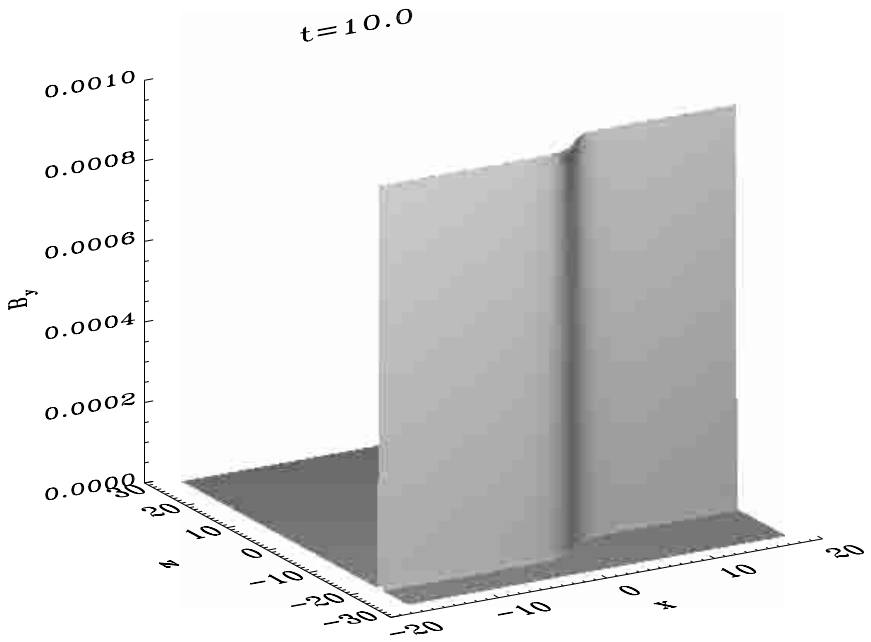

Fig. 2. Solution of the full MHD Eqs. (1)-(4) using Lare2d code, $B_{y}$, at $t=10$.

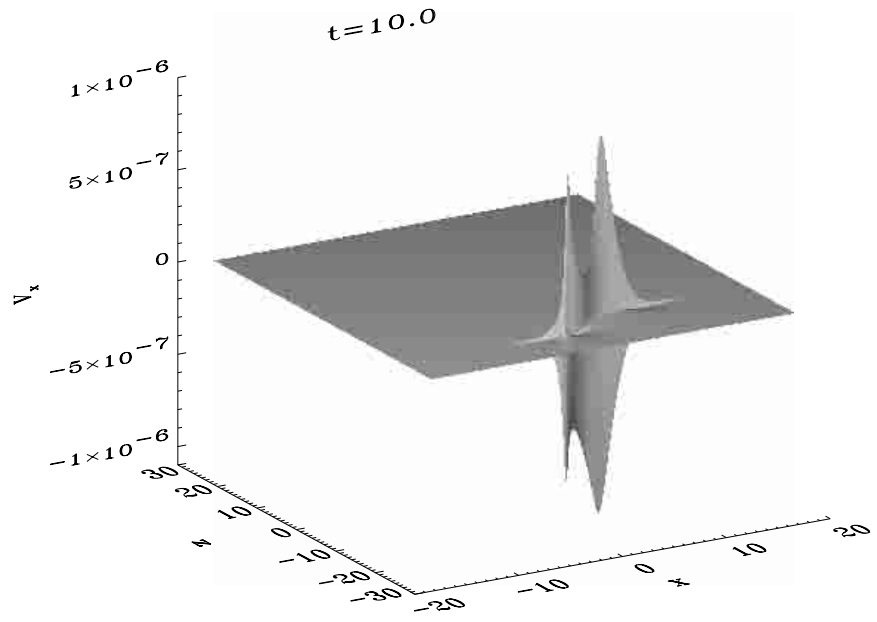

Fig. 3. Solution of the full MHD Eqs. (1)-(4) using Lare2d code, $V_{x}$, at $t=10$.

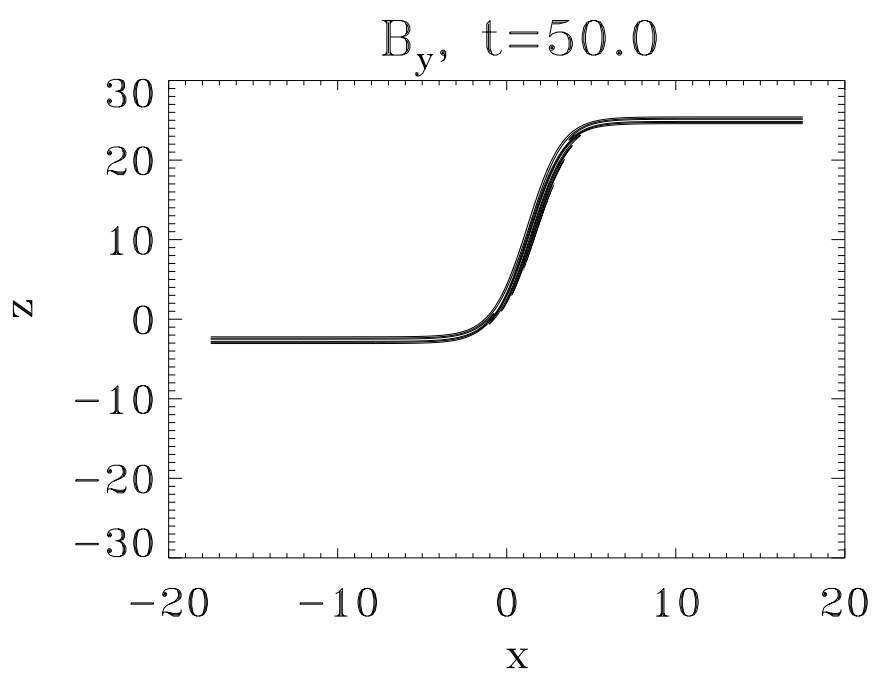

Fig. 4. Contourplot of solution of the full MHD Eqs. (1)-(4) using Lare2d code, $B_{y}$, at $t=50$. 


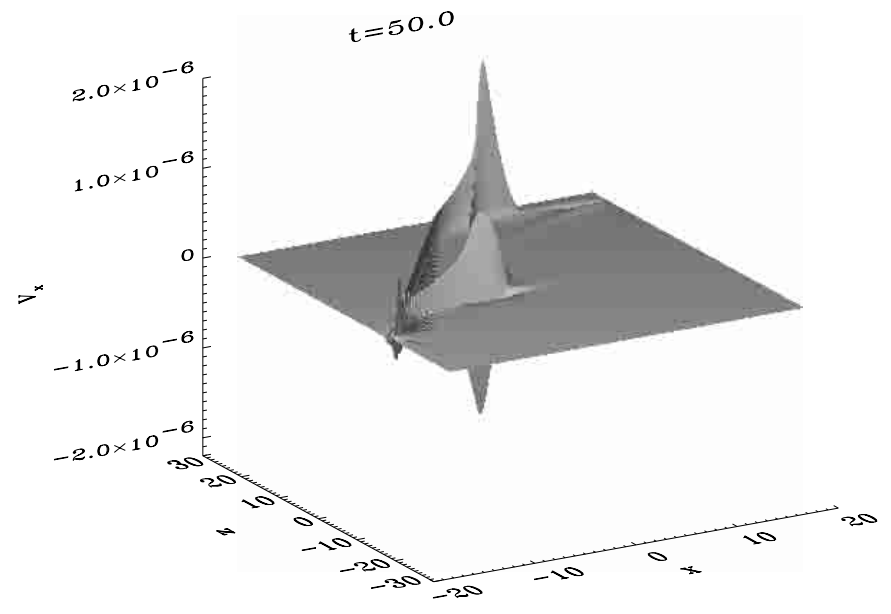

Fig. 5. Solution of the full MHD Eqs. (1)-(4) using Lare2d code, $V_{x}$, at $t=50$.

the weakly non-linear Eq. (12) directly, using a second order MacCormak scheme, for the driver given by Eq. (14). This driver can be easily written by substituting Eq. (14) into Eq. (12):

$D(x, z, t)=-\frac{2 A^{2}}{\delta} C_{\mathrm{A}}^{2}(x) \frac{\mathrm{d} C_{\mathrm{A}}(x)}{\mathrm{d} x}\left[\frac{4}{\delta}\left(z-C_{\mathrm{A}}(x) t\right)^{2} \times\right.$

$\left.C_{\mathrm{A}}(x) t+\left(z-2 C_{\mathrm{A}}(x) t\right)\right] \times \exp \left(-\frac{2\left(z-C_{\mathrm{A}}(x) t\right)^{2}}{\delta}\right)$,

where $D(x, z, t)$ is the right hand side of Eq. (12). We have solved Eq. (12) on a simulation box $-30<x<30$ and $-30<z<30$ using different spatial resolution. The results for the $V_{x}^{a}(t)$ are plotted in Fig. 6. We observe that for $4000 \times 4000$ resolution (thin solid line) the behavior of the maximal value of generated fast magnetoacoustic wave is quite similar to the results produced by the fully nonlinear Lare2d code. In Fig. 7 we plot the generated $V_{x}$ at time instance $t=50$ obtained by the 2D MacCormak code which solves Eq. (12). The similarity with Fig. 5 is obvious, which validates our earlier statement that Eq. (12) describes the dynamics of the generated fast magnetoacoustic wave to a sufficient accuracy even at later times.

As can be seen from Eq. (15) the driver of Eq. (12) contains a third order polynomial in time. Thus, one would expect continuous secular growth of $V_{x}^{a}(t)$. However, as can be seen from Fig. $6, V_{x}^{a}(t)$ at the later stages grows more slowly than initially. The situation is quite similar to observed by Botha et al. (2000). Namely, in spite of a secularly growing driver, the solution of Eq. (12) saturates. Botha et al. (2000) have speculated that the saturation of fast magnetoacoustic wave is due to the destructive wave interference effect. Thus, in order to bring clarity into the situation, following Botha et al. (2000), we also calculated the 1D analog of Eq. (12). We have solved equation

$\frac{\partial^{2} V_{x}}{\partial t^{2}}-C_{\mathrm{A}}^{2}(x) \frac{\partial^{2} V_{x}}{\partial x^{2}}=D_{1}(x, t)$

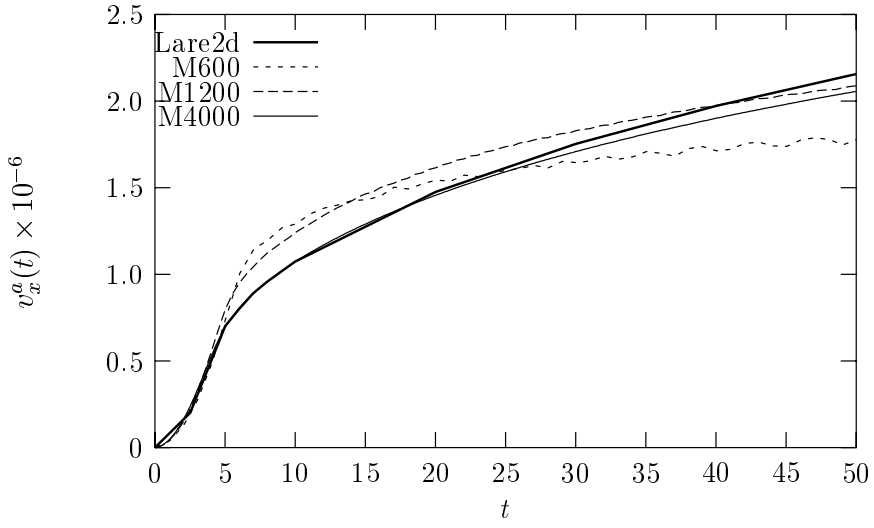

Fig. 6. Maximal value of the generated fast magnetoacoustic wave, $V_{x}^{a}(t)$, versus time. Thick solid curve represents the solution of the full MHD Eqs. (1)-(4) using Lare2d code. Thin solid, long dashed and short dashed curves represent solutions of the Eq. (12) using 2D MacCormak code on $4000 \times 4000$, $1200 \times 1200$ and $600 \times 600$ spatial resolution accordingly.

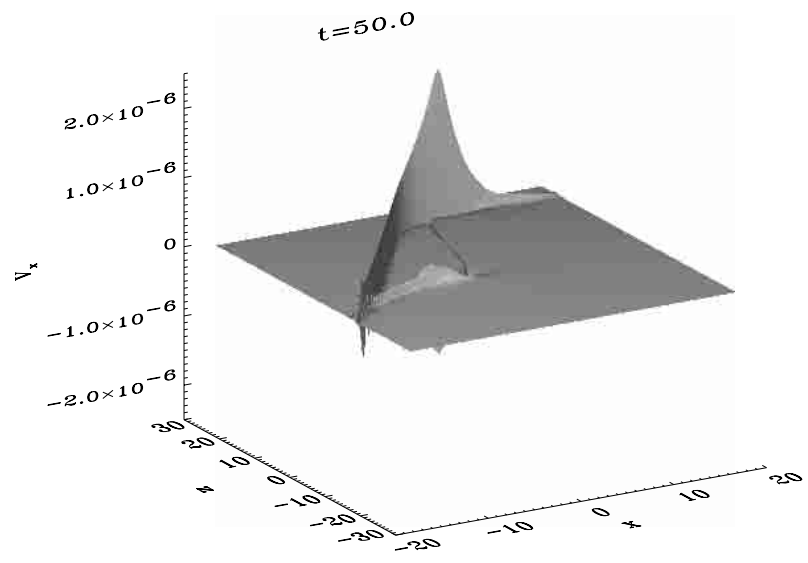

Fig. 7. Solution of the Eq. (12) using 2D MacCormak code with $4000 \times 4000$ spatial resolution, $V_{x}$, at $t=50$.

where $D_{1}(x, t)=D\left(x,\left(C_{\mathrm{A}}(+\infty)-C_{\mathrm{A}}(-\infty)\right) t, t\right)$. In other words we put $z \rightarrow\left(C_{\mathrm{A}}(+\infty)-C_{\mathrm{A}}(-\infty)\right) t$, which physically means that we reduce a $2 \mathrm{D}$ problem into a $1 \mathrm{D}$ problem by making a transformation to the frame which moves with the mid-point of phase mixed Alfvén pulse moving with velocity $\left(C_{\mathrm{A}}(+\infty)-C_{\mathrm{A}}(-\infty)\right) t$. We solved Eq. (16) with various spatial resolutions to make sure that the results converge. In Fig. 8 we plot the solution of Eq. (16) on the simulation interval $-55<x<55$ using 20000 grid points. At time $t=0 V_{x}$ is zero. The top row shows $V_{x}$ at time instances $t=1,10,50$. We see that the solution comprises two pulses moving out into positive and negative directions at speeds $C_{\mathrm{A}}(x)=1 / \sqrt{5}$ for $x<0$ and $C_{\mathrm{A}}(x)=1$ for $x>0$. The bottom row contains plots of the driver, $D_{1}(x, t)$, at the same instances. Interestingly, we observe that initially, the driver is asymmetric and has a larger positive part, however at the later stages it becomes symmetric and progressively narrow. This means that, similar to results obtained by Botha et al. (2000), 
the fast magnetoacoustic waves generated by such a driver will saturate because the positive and negative parts of the driver, which get closer as time progresses, cancel each other due to destructive wave interference. The use of the phrase "destructive wave interference" is motivated by the following reason: from wave theory we know that for two waves to compensate (to destroy) each other via interference at a given point in space, they should be $\pi$ radians out of phase. In our case the equally sized positive and negative parts of the driver move progressively closer and the waves they generate become out of phase. This interference suppresses further generation of the fast magnetoacoustic waves. The difference between our results and the ones by Botha et al. (2000) is that since we have considered a single Gaussian pulse (hoping that a single pulse will not trigger a destructive wave interference effect) we have a single symmetric driver. Botha et al. (2000) considered a harmonic initial Alfvén perturbation, and thus obtained a driver with a number of symmetric peaks, whose number grows in time (see Fig. 6 in Botha et al. 2000 for comparison). We have kept track of the maximal value of generated fast magnetoacoustic wave, $V_{x}^{a}(t)$, as our $1 \mathrm{D}$ code progressed in time. The results for the three values of $\lambda$ are presented in Fig. 9. It should be noted that there is a difference between 2D (Fig. 6) and $1 \mathrm{D}$ (Fig. 9) results. In 2D, as opposed to $1 \mathrm{D}$, we do not observe clear saturation of $V_{x}^{a}(t)$. We can only conclude from Fig. 6 that at the later stages $V_{x}^{a}(t)$ grows much slower than initially. This can be explained by the fact that in $1 \mathrm{D}$, we define $V_{x}^{a}(t)$ as a maximal value of the generated fast magnetoacoustic wave in the frame which moves with the mid-point of phase-mixed Alfvén pulse having the velocity $\left(C_{\mathrm{A}}(+\infty)-C_{\mathrm{A}}(-\infty)\right) t$. Therefore in $2 \mathrm{D}$ the quantity that would be more appropriate to compare to $V_{x}^{a}(t)$ from $1 \mathrm{D}$ results is the maximal value of generated fast magnetoacoustic wave in $1 \mathrm{D}$ slices along $x$ coordinate which are taken at $z=\left(C_{\mathrm{A}}(+\infty)-C_{\mathrm{A}}(-\infty)\right) t$ as the 2D MacCormak code progress in time. We denote the latter quantity by $V_{x}^{m}(t)=\max \left(\mid V_{x}(x, z=\right.$ $\left.\left.\left(C_{\mathrm{A}}(+\infty)-C_{\mathrm{A}}(-\infty)\right) t, t\right) \mid\right)$. In Fig. 10 we plot $V_{x}^{m}(t)$ for three values of $\lambda=0.1,0.5,2.5$. These are the same values as are used in Fig. 1. The $\lambda$-parameter controls strength of the transversal gradient. The noteworthy features that can be observed from the plot are as following: stronger transverse gradients (larger $\lambda$ 's) cause an earlier start of the fast magnetoacoustic waves saturation process, and in turn, yield lower saturation levels. Weaker gradients (smaller $\lambda$ 's) result in later onset of the saturation, and in turn, higher saturation levels. This result, actually, validates our explanation of the fast magnetoacoustic wave saturation by the destructive wave interference effect, namely, larger $\lambda$ means a steeper transverse gradient, i.e. narrower driver acting from the beginning. Therefore, destructive wave interference effect starts to a play role earlier, yielding faster shutdown in the growth of $V_{x}$. For the weaker gradients, i.e. small $\lambda$, the opposite is true. This result is in agreement with that obtained by Botha et al. (2000) (see Fig. 3 in Botha et al. 2000 for comparison). Another important
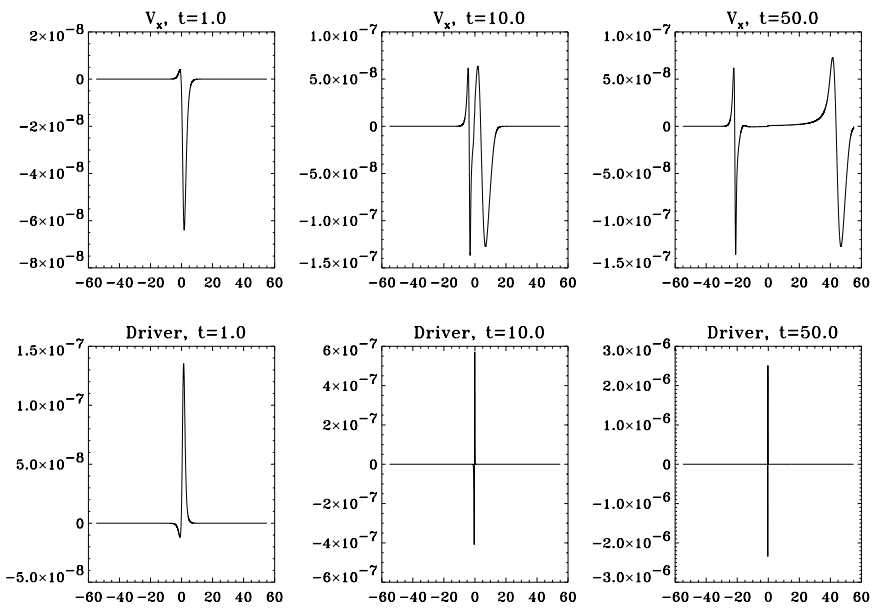

Fig. 8. Top row: solution of Eq. (16) using 1D MacCormak code on 20000 spatial resolution, $V_{x}$, at times $t=1,10,50$; bottom row: driver of Eq. (16), $D_{1}(x, t)$, at times $t=1,10,50$.

observation from Fig. 10 is that the saturation of $V_{x}^{m}(t)$ is similar to that from 1D results (Fig. 9). This differs from the results shown in Fig. 6 in which the amplitude of $V_{x}$ continues to grow albeit not at the rapid initial phase from $t=0$ to $t=5$. The discrepancy between the $1 \mathrm{D}$ and $2 \mathrm{D}$ results can be understood from the form of the $2 \mathrm{D}$ solution in Fig. 5. Here it is clear that the maximum $V_{x}$ in the box is not on the $x$ line used to produce Fig. 10 but is located at regions where the background density levels off to a constant value. At these locations the $x$ gradient of $B_{y}^{2}$ remains small but does not contract in $x$ as rapidly as in the centre of the phase-mixing region. Thus these regions are a source of continued secular growth in $V_{x}^{a}$ for longer times.

Following Botha et al. (2000) we have performed a study of parameter space, by investigating the dependence of the fast magnetoacoustic wave saturation levels upon the two free parameters in our problem, namely, $\lambda$ and $\delta$, using the 2D MacCormak code. In Fig. 11 we plot $V_{x}^{a}(t)$ for three values of $\lambda=0.1,0.5,2.5$. Again, the noteworthy features are: stronger transverse gradients (larger $\lambda$ 's) cause earlier start of the fast magnetoacoustic waves saturation process, and in turn, yield lower saturation levels. Weaker gradients (smaller $\lambda$ 's) result in later onset of the saturation, and in turn, higher saturation levels. It is worthwhile to note that the $1 \mathrm{D}$ results (Fig. 9) of the variation of $\lambda$-parameter show a similar trend, i.e. start of the saturation process earlier for larger $\lambda$ 's. However, as in the $1 \mathrm{D}$ results of Botha et al. (2000) (see p. 1191 in their paper for the discussion) the scaling of the saturation amplitudes with $\lambda$ is not the same as in $2 \mathrm{D}$ or full MHD simulations.

In Fig. 12 we investigate the scaling of saturation levels of the generated fast magnetoacoustic waves with the $\delta$-parameter, which controls width of the phase-mixed Alfvén pulse over $z$-coordinate. Figure 12 shows that with the decrease of $\delta$, i.e. for narrower Gaussian pulses, 


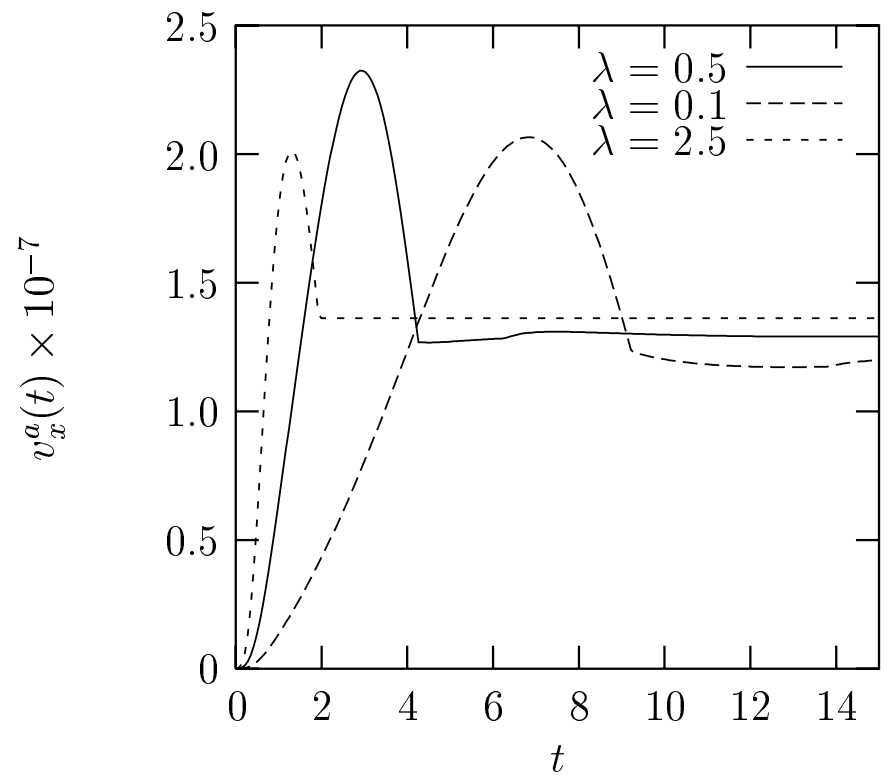

Fig. 9. Maximal value of the generated fast magnetoacoustic wave, $V_{x}^{a}(t)$, versus time. Solid, long dashed and short dashed curves represent solutions of Eq. (16) using 1D MacCormak code on 20000 spatial resolution for $\lambda=0.5,0.1,2.5$ accordingly.

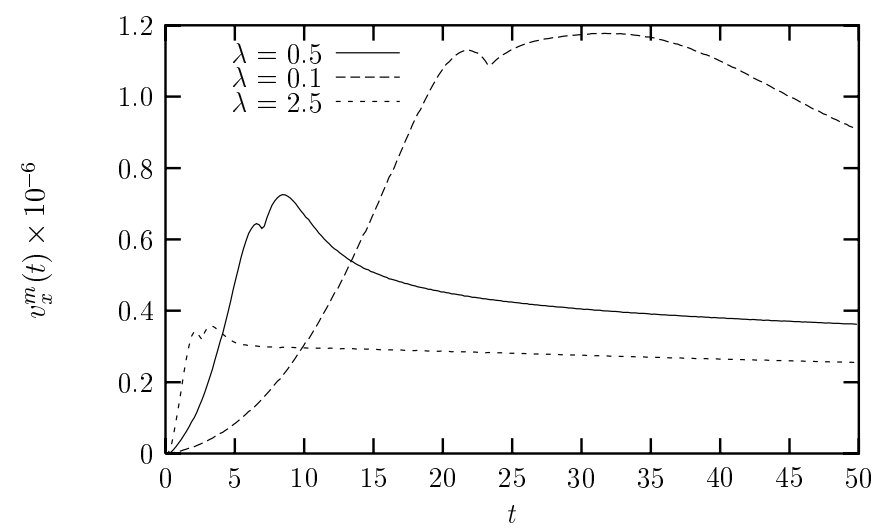

Fig. 10. Maximal value of the generated fast magnetoacoustic wave, $V_{x}^{m}(t)$, in $1 \mathrm{D}$ slices along $x$-coordinate which are taken at $z=\left(C_{\mathrm{A}}(+\infty)-C_{\mathrm{A}}(-\infty)\right) t$ versus time. Solid, long dashed and short dashed curves represent solutions of Eq. (12) using 2D MacCormak code on $4000 \times 4000$ spatial resolution for $\lambda=$ $0.5,0.1,2.5$ accordingly.

saturation levels of the generated fast magnetoacoustic waves increase and vice versa.

Finally, we would like to comment on the choice of the upper bound of our simulation time, which we have set to $t=50.0$. DeMoortel et al. (1999) have found that in linear phase-mixing (with the dissipation effects included) shear viscosity dissipation reaches its maximum when the harmonic Alfvén wave is 10 wavelengths out of phase. Assuming that this result holds for the traveling Gaussian Alfvén pulse, we can estimate the time, $t_{*}$, it takes for the classic shear viscosity to become significant.

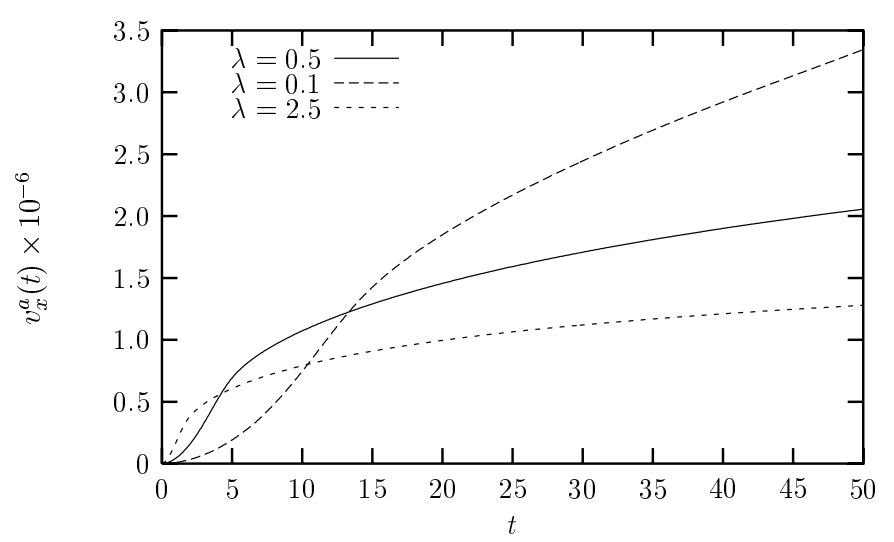

Fig. 11. Maximal value of the generated fast magnetoacoustic wave, $V_{x}^{a}(t)$, versus time. Solid, long dashed and short dashed curves represent solutions of Eq. (12) using 2D MacCormak code on $4000 \times 4000$ spatial resolution for $\lambda=0.5,0.1,2.5$ accordingly.

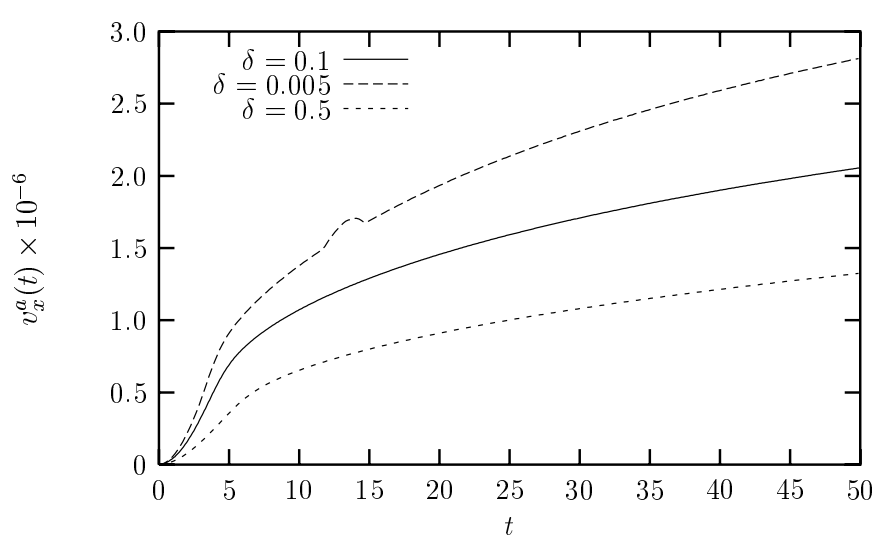

Fig. 12. Maximal value of the generated fast magnetoacoustic wave, $V_{x}^{a}(t)$, versus time. Solid, long dashed and short dashed curves represent solutions of Eq. (12) using 2D MacCormak code on $4000 \times 4000$ spatial resolution for $\delta=0.1,0.005,0.5$ accordingly.

The distance between the wings of the distorted phase mixed Alfvén pulse (see Fig. 4) is $\left(C_{\mathrm{A}}(+\infty)-C_{\mathrm{A}}(-\infty)\right) t_{*}$. If we use the pulse width, $2 \sqrt{\delta}$, instead of wavelength in the original DeMoortel et al. (1999) estimate, we can write $\left(C_{\mathrm{A}}(+\infty)-C_{\mathrm{A}}(-\infty)\right) t_{*}=10 \times(2 \sqrt{\delta})$. Inserting our values for the asymptotic Alfvén speeds and $\delta=0.1$ in the latter equality, we deduce $t_{*} \approx 11$. Therefore, there is little point running the present model for $t>t_{*}$, because as shown by DeMoortel et al. (1999), viscosity would be significant for such later times and this is excluded from our current model.

\section{Discussion}

The motivation of this paper was three-fold. Botha et al. (2000) concluded that for harmonic phase-mixed Alfvén perturbations the generated fast magnetoacoustic waves do not grow to a substantial fraction of the Alfvén wave amplitude. Firstly, we wanted to test whether a single 
phase-mixed Gaussian Alfvén pulse behaves in the same way. Secondly, we wanted to test the validity of the weakly non-linear analytic description of Nakariakov et al. (1997) for large times. Thirdly, we wanted to check whether the rapid growth (in time) of the generated fast magnetoacoustic waves still holds at the later stages.

Our present analysis has clearly demonstrated that:

- Small-amplitude, phase-mixed, single Alfvén pulses do not generate fast magnetoacoustic waves with large amplitudes. The saturation is due to the destructive wave interference.

- We have confirmed that the weakly non-linear analytic description of Nakariakov et al. (1997) (Eq. (12)) is valid and produces results that are entirely consistent with the simulation results from fully non-linear MHD Eqs. (1)-(4) at all stages, provided the initial amplitude of the Alfvén perturbation is small.

- We have found that the fast magnetoacoustic wave, generated by the phase-mixed single Alfvén pulse, at the later stages grows much slower than at earlier times, due to the destructive wave interference.

We have also established that the stronger transverse gradients (larger $\lambda$ 's) cause an earlier start of the fast magnetoacoustic waves saturation process, and in turn, yield lower saturation levels. However, weaker gradients (smaller $\lambda$ 's) result in later onset of the saturation, and in turn, higher saturation levels. This result, actually, validates our explanation of the fast magnetoacoustic wave saturation by the destructive wave interference effect. Namely, larger $\lambda$ means steeper transverse gradient, i.e. narrower driver, acting from the beginning. Therefore, the destructive wave interference effect starts to a play role earlier yielding faster shutdown in the growth of $V_{x}$. For the weaker gradients, i.e. small $\lambda$, exactly the opposite is true. This result is in agreements with that obtained by Botha et al. 2000 for a harmonic Alfvén wave (see Fig. 3 in Botha et al. 2000 for comparison). It is worth mentioning, however, that contrary to the one's expectations, steeper inhomogeneities of the Alfvén speed do not provide higher saturation levels of the fast wave generation. In other words, with the increase of $\lambda$, saturation levels decrease. On the other hand, in the homogeneous plasma $(\lambda=0)$ there is no generation of the fast magnetoacoustic wave by a plane (with respect to $x$-coordinate) Alfvén wave at all, i.e. $V_{x}^{a}(t) \rightarrow 0$ when $\lambda \rightarrow 0$. Therefore, there is a certain optimal value of the gradient of the inhomogeneity for which the fast wave saturation level is maximal. At this stage we have not performed a full parametric study in order to determine the optimal value of $\lambda$ due to the huge requirements for $\mathrm{CPU}$ time.

The mechanism of indirect plasma heating by the fast magnetoacoustic waves that are non-linearly generated by the phase-mixed Alfvén perturbation, discussed in this paper, is certainly applicable for the coronal heating problem through dissipation of fast magnetoacoustic waves in open (e.g. plumes) and closed (loops) coronal plasma structures.
We found that the proposed mechanism, in the considered set up, is limited by the destructive wave interference. Thus, further work is needed to find possible ways around the encountered difficulty. Possible cures might be to consider $2 \mathrm{D}$ density inhomogeneity profiles that would prevent the driver of Eq. (12) from becoming too narrow, and/or inclusion of the dissipation into our model, that would prevent the transverse scale collapse, which all ultimately will lead to the decrease of the destructive wave interference.

Acknowledgements. Numerical calculations of this work were done using the PPARC funded Compaq MHD Cluster in St. Andrews. DT acknowledges financial support from PPARC Postdoctoral Fellowship.

\section{References}

Abdelatif, T. E. 1987, ApJ, 322, 494

Arber, T. D., Longbottom, A. W., Gerrard, C. L., \& Milne, A. M. 2001, J. Comput. Phys., 171, 151

Botha, G. J. J., Arber, T. D., Nakariakov, V. M., \& Keenan, F. P. 2000, A\&A, 363, 1186

Browning, P. K. 1991, Plasm. Phys. Contr. Nucl. Fus., 33, 539

Browning, P. K., \& Priest, E. R. 1984, A\&A, 131, 283

De Moortel, I., \& Hood, A. W. 2000, A\&A, 363, 269

DeMoortel, I., Hood, A. W., Ireland, J., \& Arber, T. D. 1999, A\&A, 346, 641

De Moortel, I., Hood, A. W., \& Arber, T. D. 2000, A\&A, 354, 334

Grappin, R., Leorat, J., \& Buttighoffer, A. 2000, A\&A, 362, 342

Heyvaerts, J., \& Priest, E. R. 1983, A\&A, 117, 220

Hood, A. W., Ireland, J., \& Priest, E. R. 1997, A\&A, 318, 957

Ireland, J. 1996, Ann. Geophysic., 14, 485

Ireland, J., \& Priest, E. R. 1997, Sol. Phys., 173, 31

Malara, F., Primavera, L., \& Veltri, P. 1996, ApJ, 459, 347

Nakariakov, V. M., Ofman, L., \& Arber, T. D. 2000, A\&A, 353,741

Nakariakov, V. M., Ofman, L., DeLuca, E. E., Roberts, B., \& Davila, J. M. 1999, Science, 285, 862,

Nakariakov, V. M., Roberts, B., \& Murawski, K. 1997, Sol. Phys., 175, 93

Nakariakov, V. M., Roberts, B., \& Murawski, K. 1998, A\&A, 332,795

Nocera, L., Priest, E. R., \& Hollweg, J. V. 1986, Geoph. Astrophys. Fluid Dyn., 35, 111

Ofman, L., \& Davila, J. M. 1997, ApJ, 476, 357

Parker, E. N. 1991, ApJ, 376, 355

Roberts, B. 2000, Sol. Phys., 193, 139

Roberts, B. 1991, in Advances in Solar System Magnetohydrodynamics, ed. E. R. Priest, \& A. W. Hood (Cambridge Univ. Press)

Roberts, B., \& Ulmschneider, P. 1997, in Solar and Heliospheric Plasma Physics, ed. G. Simnett et al. (Springer)

Ruderman, M. S., Goldstein, M. L., Roberts, D. A., Deane, A., \& Ofman, L. 1999, JGR, 104, 17057

Ruderman, M. S., Nakariakov, V. M., \& Roberts, B. 1998, A\&A, 338, 1118

Ryutova, M. P., \& Habbal, S. R. 1995, ApJ, 451, 381

Sakurai, T., \& Granik, A. 1984, ApJ, 277, 404

Tsiklauri, D., Nakariakov, V. M., \& Arber, T. D. 2001, A\&A, submitted 